\title{
Nécrose graisseuse épipéricardique
}

\section{Epipericardial Fat Necrosis}

\author{
P. C. Thiebaud $\cdot$ A. Muret $\cdot$ F. Brigant
}

Reçu le 23 février 2018; accepté le 4 avril 2018

(C) SFMU et Lavoisier SAS 2018
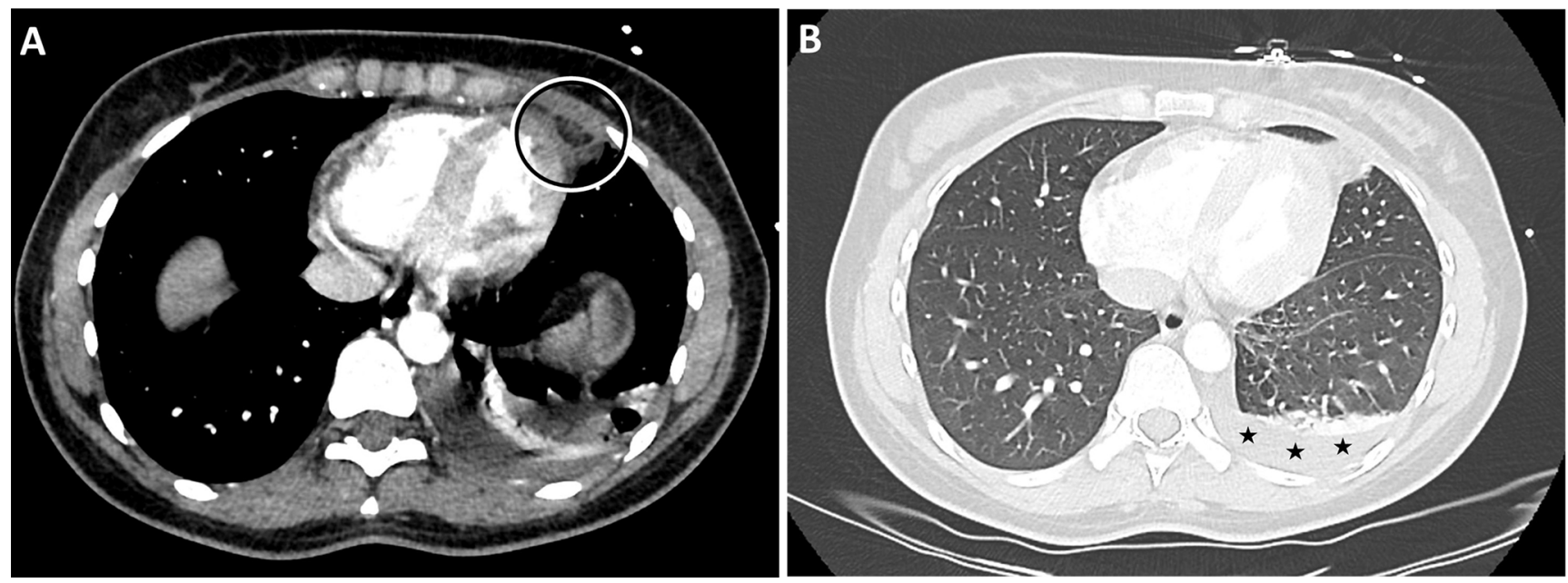

Fig. 1 Angioscanner thoracique en coupe axiale. A. Fenêtre médiastinale : lésion ovoïde de densité centrale graisseuse en regard de la pointe du ventricule gauche (cercle) évoquant une nécrose de frange graisseuse épipéricardique. B. Fenêtre parenchymateuse : épanchement pleural gauche de moyenne abondance (étoiles)

Une femme de 24 ans sans antécédent médico-chirurgical est adressée aux urgences par son médecin traitant pour suspicion d'embolie pulmonaire. Elle présente une douleur basithoracique antérieure gauche d'apparition progressive depuis 5 jours, sans signe associé. Un bilan biologique réalisé en ville retrouve des d-dimères positifs à $1640 \mu \mathrm{g} / \mathrm{L}$ et l'absence de syndrome inflammatoire biologique significatif (5400 leucocytes, CRP $24 \mathrm{mg} / \mathrm{L}$ ). Une radiographie thoracique de face montre des images d'allure alvéolaires au niveau du lobe inférieur gauche, sans foyer infectieux ni épanchement pleural identifié. Aux urgences, il existe une tachycardie à 120/min, les autres paramètres vitaux étant normaux (pression artérielle 139/83 $\mathrm{mmHg}$, fréquence respiratoire $16 / \mathrm{min}$, saturation en oxygène $100 \%$ en air

\footnotetext{
P. C. Thiebaud $(\triangle) \cdot$ A. Muret $\cdot$ F. Brigant

Service des urgences, Hôpital Saint-Antoine,

Assistance Publique-Hôpitaux de Paris (AP-HP),

184, rue du Faubourg Saint-Antoine, F-75012 Paris, France

e-mail : pc.thiebaud@hotmail.fr
}

ambiant, température $37,2^{\circ} \mathrm{C}$ ). La douleur basithoracique gauche est spontanément présente et majorée à l'inspiration profonde. Le reste de l'examen clinique est normal. L'électrocardiogramme (ECG) montre une tachycardie sinusale, un aspect de bloc de branche droit, sans autre anomalie de conduction ou de repolarisation. Un angioscanner thoracique est alors réalisé (Fig. 1). Il élimine l'embolie pulmonaire et montre une nécrose de frange graisseuse épipéricardique associée à un épanchement pleural gauche de moyenne abondance. Il existe un collapsus pulmonaire passif au contact de l'épanchement pleural, qui pourrait expliquer les images alvéolaires décrites sur la radiographie initiale. La douleur disparait rapidement après initiation d'un traitement par anti-inflammatoires non stéroïdiens (AINS), ce qui permet un retour à domicile de la patiente. Un scanner de contrôle réalisé 1 mois plus tard montre la disparition de la nécrose graisseuse épipéricardique et de l'épanchement pleural.

La nécrose de la graisse épipéricardique est une cause rare de douleur thoracique, bénigne et probablement 
sous-diagnostiquée $[1,2]$. Elle se présente le plus souvent par l'apparition brutale d'une douleur thoracique d'allure pleurale, sans signe associé ni facteur déclenchant identifié, chez un sujet jeune en bonne santé. Elle est plus fréquente du côté gauche et habituellement associée à un épanchement pleural homolatéral, qui pourrait être à l'origine de la douleur thoracique. L'examen clinique est souvent normal et il n'existe pas d'anomalie spécifique sur l'ECG ou le bilan biologique. La radiographie thoracique est peu contributive mais peut montrer l'épanchement pleural. C'est le scanner qui fait le diagnostic, en montrant une lésion arrondie ou ovoïde de densité centrale graisseuse entourée d'une pseudo-capsule dense et régulière. L'évolution est habituellement favorable avec régression rapide de la douleur sous antalgiques et AINS, et résolution des anomalies radiologiques. Aucun cas de récidive n'a été décrit dans la littérature. Le mécanisme physiopathologique n'est pas clairement identifié.

\section{Références}

1. Giassi KS, Costa AN, Bachion GH, et al (2016) Epipericardial fat necrosis: who should be a candidate? AJR Am J Roentgenol 207:773-7

2. Neuville M, Taillé C, Debray MP, et al (2015) Nécrose de la graisse médiastinale. Rev Mal Respir 32:279-82 